\title{
Improved imaging at Thunder Horse South with joint surface and borehole seismic tomography
}

Olga Zdraveva, Gloria Arango Solano, Syed Omar Zaman, Emmanuel Saragoussi, Aurora Rodriguez Castelan, WesternGeco; Samarjit Chakraborty, Qingsong Li, Corey Joy, Ken Hartman and Anya Reitz, BP

Copyright 2019, SBGf - Sociedade Brasileira de Geofísica

This paper was prepared for presentation during the $16^{\text {th }}$ International Congress of the Brazilian Geophysical Society held in Rio de Janeiro, Brazil, 19-22 August 2019.

Contents of this paper were reviewed by the Technical Committee of the $16^{\text {th }}$ International Congress of the Brazilian Geophysical Society and do not necessarily represent any position of the SBGf, its officers or members. Electronic reproduction or represent any position of the SBGf, its officers or members. Electronic reproduction of
storage of any part of this paper for commercial purposes without the written consent storage of any part of this paper for commercial
of the Brazilian Geophysical Society is prohibited.

\begin{abstract}
We present the most-recent effort in fine-tuning the earth model at the Thunder Horse producing field initiated because of, and in parallel with, the 2015 ocean-bottom node survey acquisition. To minimize uncertainty in the new earth model, the chosen approach relies on using all available information. This entails incorporating six surface seismic surveys with differing geometries, five 3D vertical seismic profile (VSP) surveys, three walkaway VSPs, five zero-offset VSPs, multiple sonic logs, and welldepth markers in the earth model building process to derive a single anisotropic model that adequately minimizes misfit for all inputs. To achieve this, we use joint tomography of surface and borehole seismic data with simultaneous updates of model parameters, including the VSP transit times and well-depth markers as a data constraint. This strategy resulted in improved surface and borehole seismic images that better resolve structural complexity and stratigraphic details, yielding a more-accurate interpretation and enabling reliable well placement plans.
\end{abstract}

\section{Introduction}

Thunder Horse South (THS) is part of a larger producing field located in the south-central part of the Mississippi Canyon protraction area of the Gulf of Mexico. This structural trap, partially covered by a salt canopy and segmented by a complex system of faults, represents a salt-withdrawal-related four-way dip closure. Oil is contained within three middle-lower Miocene units with high reservoir pressures and temperature. Since the THS discovery in 1999, a unique variety of data has been acquired to help geoscientists understand better the reservoir and the overall geologic model in the area (Pfau et al., 2002; Ray et al., 2003; Hartman et al., 2015; Reitz and Hartman, 2017).

The 2015 acquisition of a large ocean-bottom node (OBN) survey (Chakraborty et al., 2017) created a new opportunity to revise and fine-tune the existent earth model by incorporating not only the newly acquired surface seismic data, but also all legacy vertical seismic profile (VSP) surveys and well-depth markers in a joint common image point (CIP) tomography (Woodward et al.,
2008) model updating scheme. We first present the necessary quality control and data preparation steps required to ensure that the different data sets to be used in joint tomographic inversion carry the same information in regard to the earth model properties. Secondly, we discuss using joint tomography of surface seismic and borehole seismic data with a simultaneous parameter update (Zdraveva et al., 2017) in this project and how it complements the overall earth model building (EMB) strategy. Finally, we show that the new earth model yields improved borehole and surface seismic images over THS that better-resolve the structural and stratigraphic details and, therefore, represent a better starting point for future interpretation, and fine-tuning THS field-development plans.

\section{Data analysis and model validation}

There are five vintages of 3D VSP data, three walkaway (WA) VSPs (Figure 1), and five zero-offset (ZO) VSPs available in the Thunder Horse area. Early analysis of the legacy first-arrival picks' quality showed that many have apparently chaotic behavior and are not sufficiently accurate for potential use in any joint tomography inversion scheme. Repicking the first-arrival (or transit) times for the VSP data was out of the scope of this imaging exercise; instead, they were QC'ed thoroughly and editted with the obviously erroneous picks, mostly associated with wave-propagation paths through salt, excluded from further analysis.

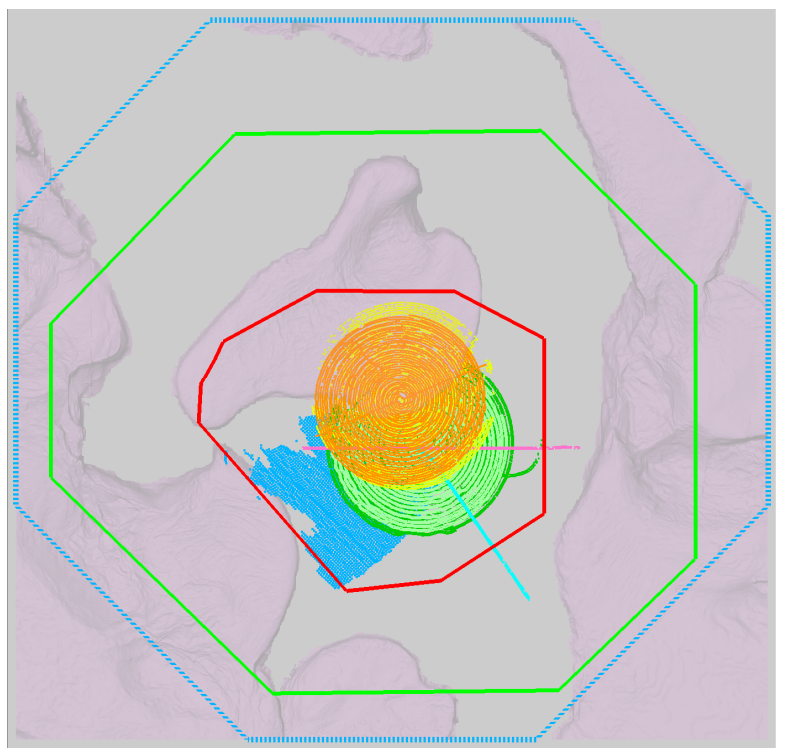

Figure 1 - A map view of post-editing source locations for the five $3 D$ VSPs and the three WA VSPs on the 
background of the salt geobody with the polygons of node locations (in red), source locations for OBN data (in green), and the towed-streamer data spread (in blue).

We decided to use all VSPs in the initial model validation stage at the beginning of the project. As discussed by Zdraveva et al. (2017), model validation is the quality control and assurance step carried out after any modification of model parameters, either in the initial model building phase or during the iterative tomography or full-waveform inversion (FWI) loop of the EMB process. In cases with no well control, the main criteria used are the model's ability to produce: (1) well-focused seismic images with minimum residual curvature on gathers, (2) geologically plausible images, and (3) model property fields that are free of artifacts and consistent with rock physics and geomechanics. In cases with well control or any other additional measurements (e.g., gravity or electromagnetic data), we add an additional criterion: (4) results consistent with well (or any other) measurements. Depending on the nature of the available well information, the consistency is quantified through any of the following: simple visual comparisons of well logs and property fields, well markers mis-tie analysis, VSP transit-time (TT) misfit graphs and maps, and examining 3D VSP images and gathers.

To calculate the TT misfit for a given model, we first simulate the source-to-receiver TTs corresponding to the actual VSP geometry using a high-accuracy $3 \mathrm{D}$ tilted transversely isotropic (TTI) two-point ray tracer. Next, we compare the simulated TT to the TT picked from the real borehole seismic data, creating a difference measurement, called the TT misfit. This measurement quantifies the model accuracy because the TT misfit will be zero if the model is sufficiently accurate and everything else in the imaging system is in balance.

The existent TTI model is a result of a previous highquality imaging exercise using three narrow-azimuth and two orthogonal wide-azimuth surveys (Hartman et al., 2015). This model produces high-quality images, reasonably flat gathers for all azimuths and close to zero mis-tie at all available well-marker control points. However, the model is inconsistent with the transit times measured in all VSP surveys as shown in Figure 2.

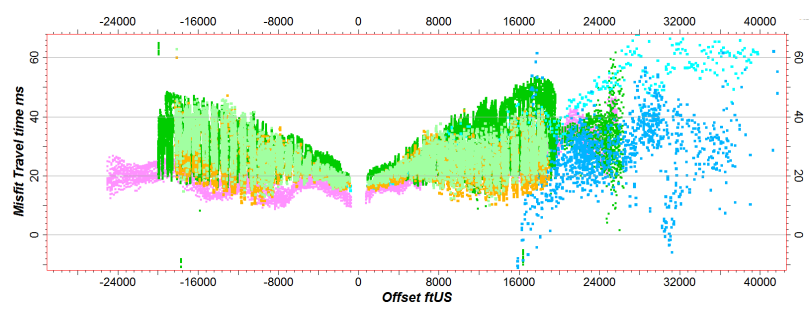

Figure 2 - TT misfit as a function of wellbore-to-source distance for the VSPs shown in Figure 1. Different colors denote different VSPS.

There is an observable bias of more than $20 \mathrm{~ms}$ in the TT misfit for all VSPs, suggesting that either the velocities are too slow or anisotropy is too high. The presence of systematic TT misfit bias was confirmed while analyzing the available five ZO VSPs. To understand the situation better, we conducted localized CIP tomography (Bakulin et al., 2010a) at Well 822-3 - the only well with a ZO VSP that is entirely outboard the salt. By using the preprocessed towed-streamer data and ZO offset VSP transit times jointly, it converged to a geologically plausible model that explained the VSP and seismic data (Figure 3).
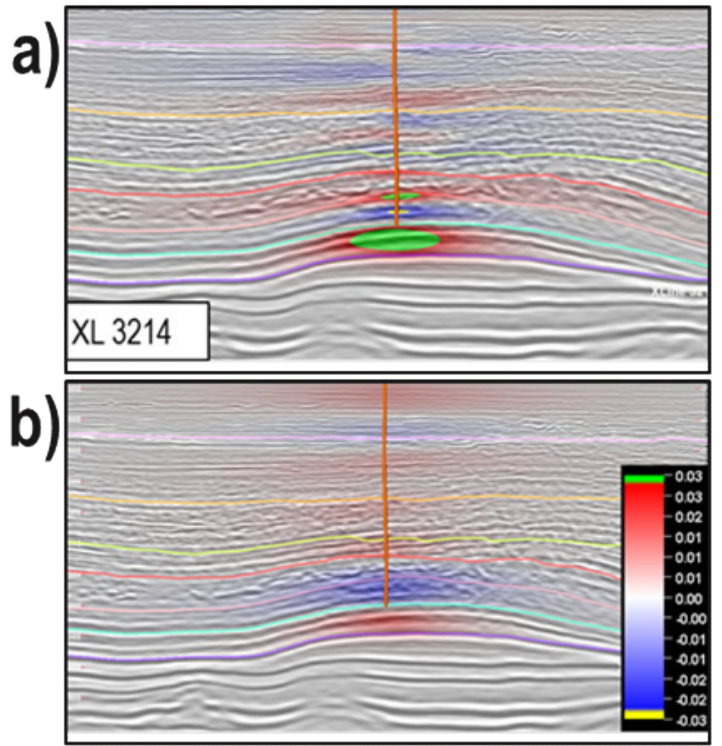

C)
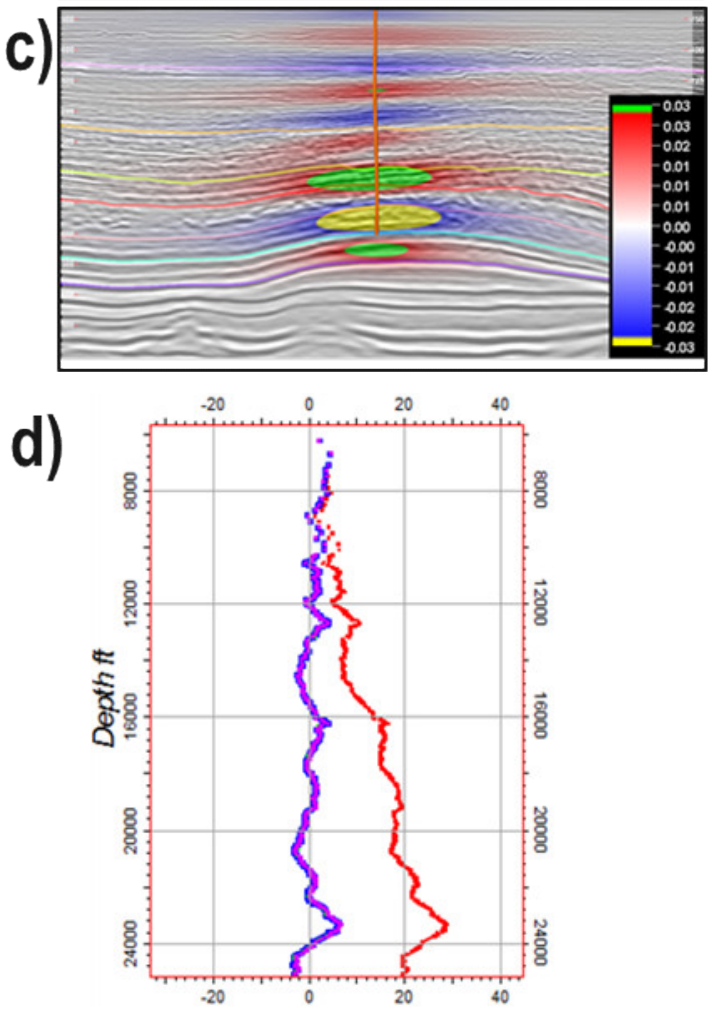

Figure 3 - Localized tomography results: seismic cross section through the Well 822-3 location with overlaid updates from the localized tomography: a) $\Delta V p$, b) $\Delta \varepsilon$, c) $\Delta \delta$, and d) TT misfit for the legacy model (red) and the models from the joint tomography iterations (in blue and pink). 
However, the corresponding image did not tie the provided well synthetics and corresponding well-depth markers. One possible explanation for such mismatch is the attenuation compensation used in the surface seismic data preprocessing, as it may imply a different reference frequency compared to the one represented by VSP transit times and well markers. A proof-of-concept study suggested that one way to explain all three sets of data simultaneously is by applying $Q$ compensation with a much higher $(250 \mathrm{~Hz}$ instead of previously used $30 \mathrm{~Hz}$ ) reference frequency for the surface seismic data. To ensure consistency between different image vintages and simplify the transition between CIP tomography and FWI iterations required for this project, we decided to keep the data reference frequency unchanged and modify the VSP transit times using Futterman's equation (Futterman, 1962) instead.

\section{Joint tomography with simultaneous updates and the production EMB strategy}

In preparation for production EMB with the new OBN data, we conducted another proof-of-concept study to demonstrate the effectiveness of joint tomography of borehole and surface seismic data with simultaneous parameter updates (Zdraveva et al., 2017) in a global sence. We used one ZO, two WA and one 3D VSP at Well 822-3. The joint tomographic update produced reduced VSP TT misfit and created a geologically plausible texture of $\delta$ and $\varepsilon$ fields in the VSP data illumination cone, similar to the corresponding updates from the localized tomography at Well 822-3 shown on Figures $3 b$ and $3 c$, but naturally extended to a larger area.

The results were compared against those from 3D VSP transit-time inversion (Leany, 2008; Li et al., 2015), which represents the current industry standard for model calibration and anisotropic property updates with 3D and WA VSP data. As expected, both solutions explained adequately the VSP data, yielding comparable close-tozero TT misfit. However, the VSP transit-time inversion, which does not use surface seismic data, fails to produce a model that can explain the surface seismic data and yield flat CIP gathers (Figure $4 b$ ).

During the project's production stage, we conducted joint tomography with simultaneous updates at multiple steps of the EMB process. First, it was used during the sediment CIP tomography following an initial pass of FWI with the new ultra-large-offset OBN data. In these updates, surface seismic data were represented by multiparameter residual moveout picked on Kirchhoff depth migration (KDM) offset gathers for four azimuths of towed-streamer data and mirror-migrated downgoing wavefield information from the new OBN data. Borehole seismic data consisted of the transit times for five 3D VSPs, three WA VSPs, and one ZO VSP.

Following salt interpretation and a deeper-section FWI update, joint tomography with simultaneous updates was conducted again using reverse time migration (RTM) subsurface-azimuth angle gathers (Vyas et al., 2011), as well as all interpreted, mostly subsalt, well-marker depths, together with the VSP transit times as a constraint. In addition, in the area of the known existing oil-water contact (OWC) at THS, the well-marker control points were extended to a full horizon patch. Using a horizon patch, as opposed to isolated points where the well paths are located, is possible in this case because of the predictable shape of the OWC surface.

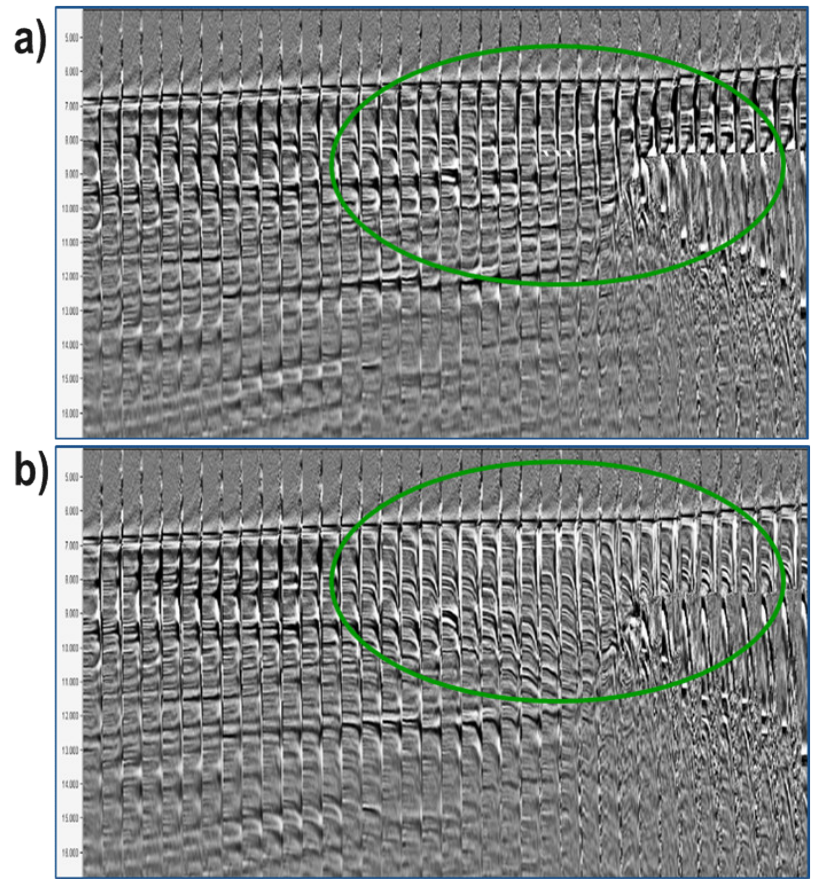

Figure 4 - CIP gathers along a line passing through the THS area: a) model updated with joint tomography, and b) model updated using the $3 D$ VSP transit times only.

All CIP tomography runs used structure-guided solution shaping with steering filters (Bakulin et al., 2010b) and were followed by thorough model validation using all required and described earlier criteria.

\section{Final model validation and results}

We created several sets of images from both surface and borehole seismic data with the final 2017 TTI earth model using KDM and RTM algorithms. Figure 5 compares downgoing field mirror-migration RTM images produced with the two models. It is evident that the image corresponding to the 2017 model (Figure 5b) shows improved resolution, focusing of faults, and continuity of the events above and at the crest of the structure, and a more geologically plausible shape of the base of the structure.

The residual curvature observed on the surface-azimuth offset and subsurface-azimuth angle gathers is minimal and the existing differences across the azimuths are likely due to genuine azimuthal variations caused by directional stress around the salt bodies. Accounting for these azimuthal variations in future imaging exercises may require using a lower class of anisotropy, such as orthorhombic. 


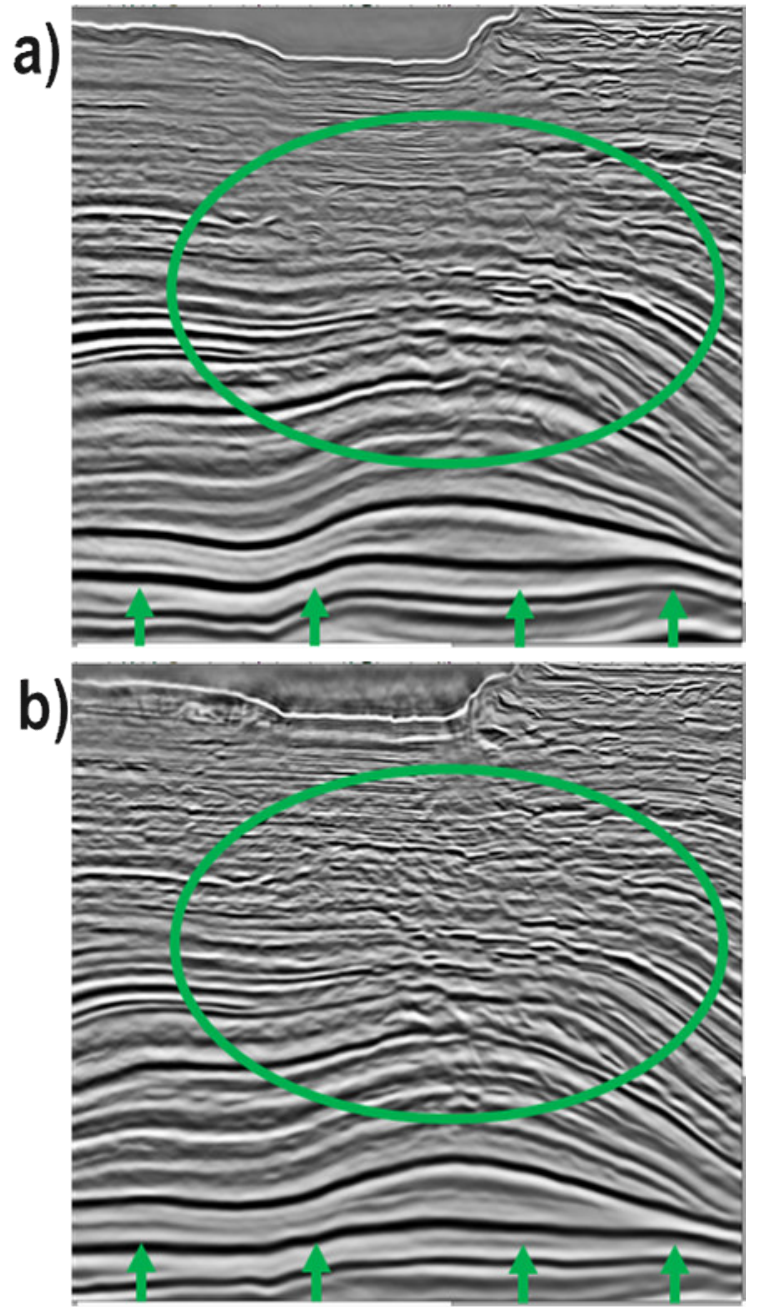

Figure 5 - RTM mirror images over THS: a) cross section from the fast-track volume migrated with the legacy TTI model, b) same cross section from the final volume using the 2017 TTI model.

The well-marker mis-ties are satisfactory and the VSP TT analysis indicates that the magnitude of the model 2017 TT misfit is generally reduced for all VSPs. When analysing TT from 3D VSPs with spiral shooting, such as the one at Well 822-3, it is additionally insightful to display the TT misfit graphs as a function of azimuth (Figure $6 a$ and $6 \mathrm{~b}$ ) to emphasize the eventual azimuthal component of the misfit, even when some of the far offset contributions for certain angles might be missing due to editing paths passing through salt (Figure 6c).

While the misfit graph corresponding to the legacy model (Figure 6a) clearly shows an apparent azimuthal variation with slow direction at approximately $160^{\circ}$, it is evident that the variation is reduced and barely observable on the model 2017 misfit graph (Figure 6b) and all points are more centred around zero.

On this project, we had the rare opportunity to conduct a validation with a blind test, as in 2017 a new 3D VSP (Well 822-14) was shot over THS field with objectives to reduce the risk of drilling sub-economic wells and potentially add new targets in the Brown and Peach reservoirs (Joy et al., 2018). As expected for a VSP shot over the same area, TT misfit graphs for the Well 822-14 $3 \mathrm{D}$ VSP that were not used in the joint tomographic updates, show similar behaviour as these of the Well 8223 3D VSP shown on Figure 6.
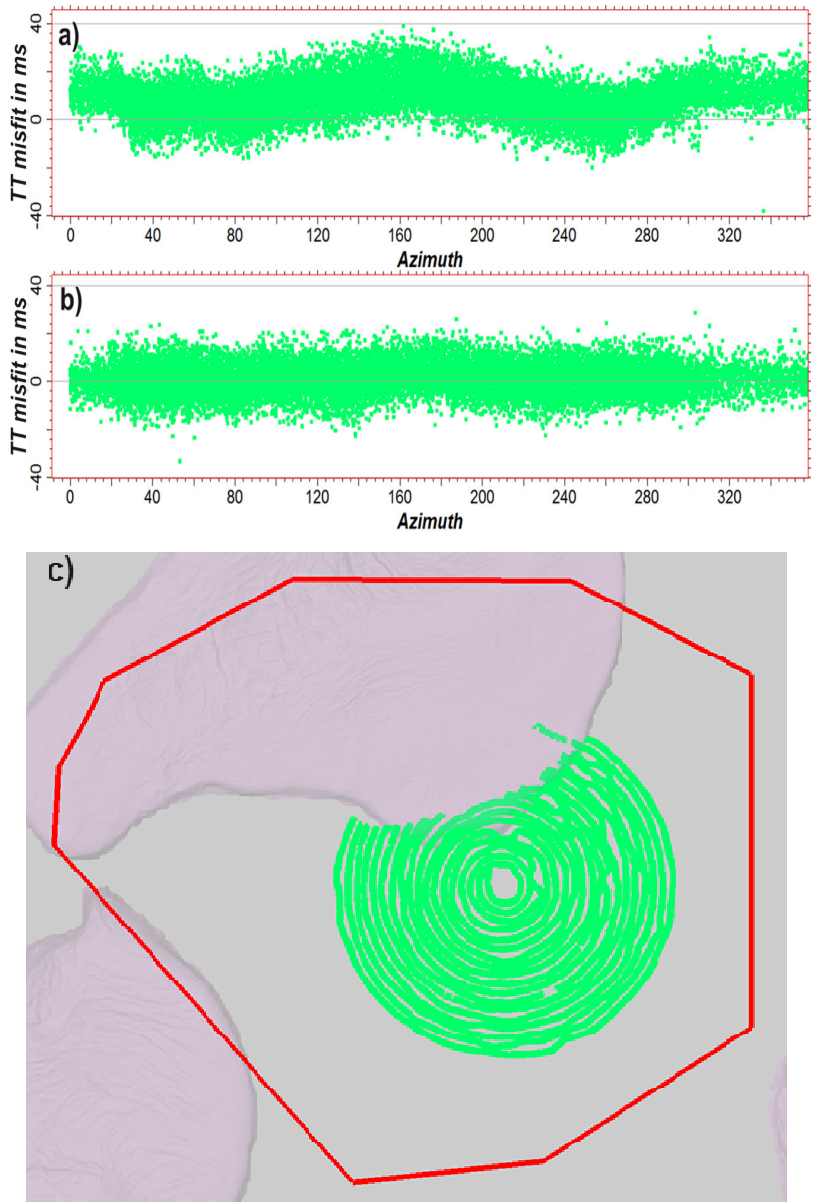

Figure 6 - TT misfit as a function of azimuth for the $3 D$ VSP at Well 822-3 - a) for the legacy model, b) for the 2017 model, and c) a map view of the $3 D$ VSP sourse locations. Note that many of the sources above the salt body (in pink) are edited out. The red polygon indicates the node locations.

Just for validation purposes, we created RTM images for all of the 3D VSPs with the legacy and 2017 models. For all the VSPs, images produced with the 2017 model show similar improvements as the surface seismic image, including the structural changes most obvious at the crest and bottom of the turtle structure. Figure 7 shows an example of raw RTM images (Figures $7 a$ and $7 \mathrm{~b}$ ) and receiver gathers (Figure $7 \mathrm{c}$ ) for the new 3D VSP at Well 822-14. Comparing RTM receiver gathers, one can clearly see that the gathers produced with the 2017 model (Figure 7c, right) are much flatter than those produced with the legacy model (Figure 7c, left) showing an upward tilt left-to-right, corresponding to shallow to deeper receiver. 

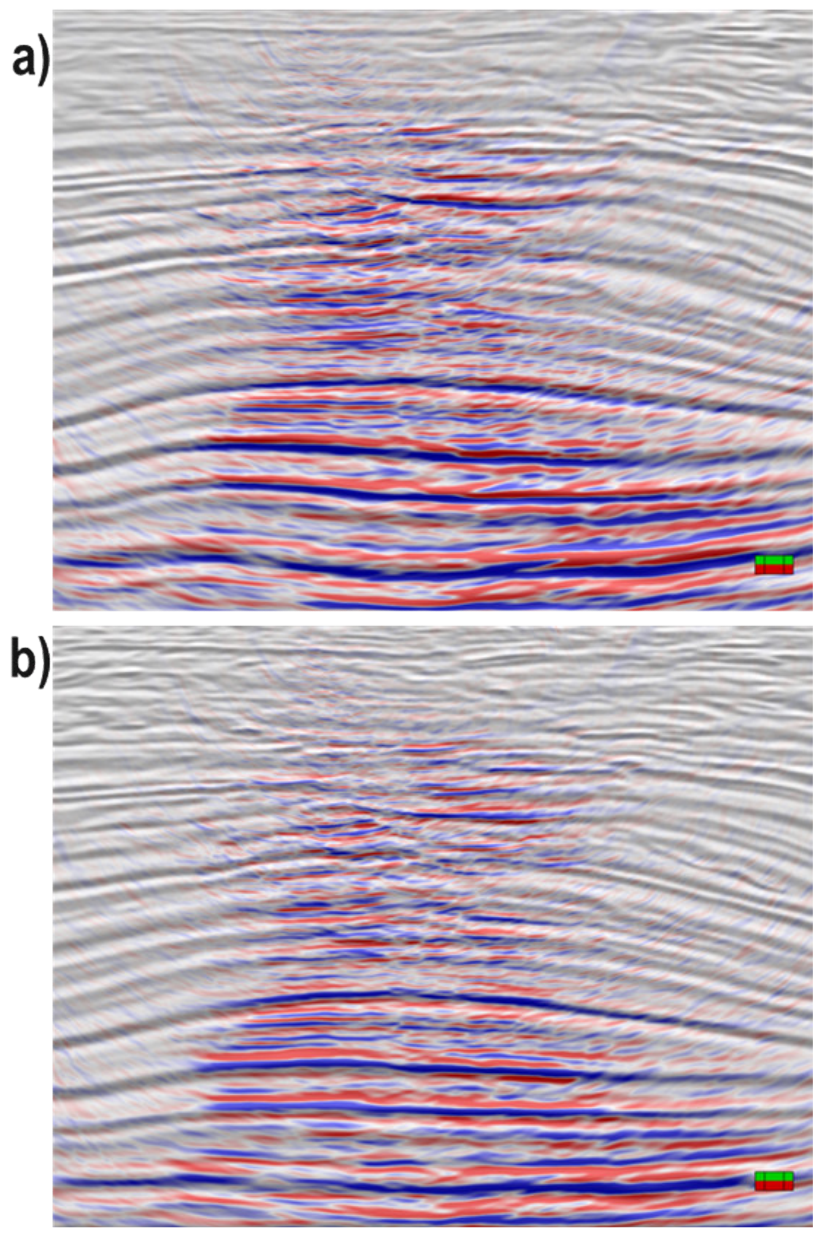

c)
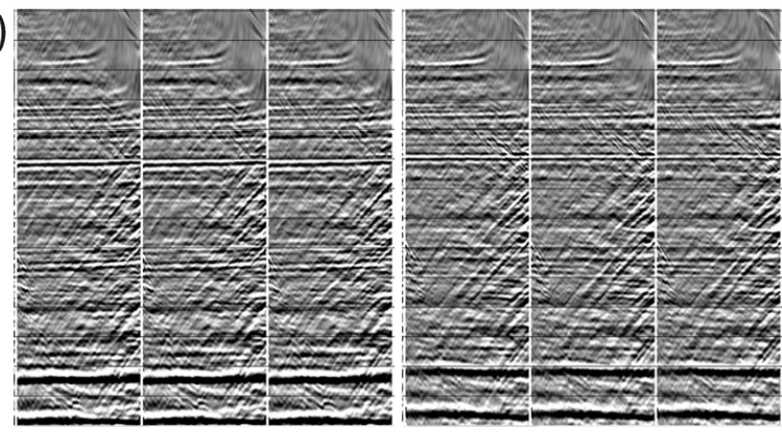

Figure 7 - Raw RTM VSP image (in blue-red scale) for Well 822-14 overlaid on OBN image (grey scale) - a) with legacy model, b) with model 2017, and c) RTM VSP receiver gathers at a location close to the well bore with legacy model (left) and 2017 model (right).

Examining all validation criteria showed that the 2017 model explains all currently available data and produces high-quality geologically plausible images for both surface seismic and borehole seismic data. The use of highly accurate models is critically important in 3D VSP imaging, especially when VSP images are expected to fill illumination gaps and provide a much higher-resolution images required for identifying and interpreting thin sand beds generally undetectable on surface seismic data. The new 2017 model yielded high-resolution VSP images of exceptional quality and enabled Joy et al. (2018) to fully achieve the Well 822-14 3D VSP objectives. An additional factor in improving the interpretability of both surface- and borehole seismic images was the extensive image enhancement efforts, involving various techniques, described by Zaman et al. (2018).

\section{Conclusions}

By conducting joint tomography with simultaneous property updates, we successfully incorporated six different inputs in the Thunder Horse EMB workflow: legacy towed-streamer and new OBN surface seismic data, legacy borehole seismic data represented by 3D, WA and ZO VSPs of various vintages, and interpreted well-marker information. The chosen strategy resulted in a high-fidelity earth model with increased accuracy, better explaining all currently available borehole information, and surface seismic data and providing a reliable baseline for future time-lapse reservoir monitoring seismic surveys. Final surface and borehole seismic images are of superior quality to any previously created images over THS. This helps interpreters understand the reservoir better, improve the quality of the interpretation products, and optimize further the production.

\section{Acknowledgements}

We thank BP, ExxonMobil, and WesternGeco for permission to publish this work and many colleagues from the Thunder Horse team for their valuable contribution.

We also thank TGS and WesternGeco for the towedstreamer data used in this work.

\section{References}

BAKULIN, A., WOODWARD, M., NICHOLS, D., OSYPOV, K., ZDRAVEVA, O. Building tilted transversely isotropic depth models using localized anisotropic tomography with well information. Geophysics, 75, D27D36, 2010a.

BAKULIN, A., WOODWARD, M., LIU, Y., ZDRAVEVA, O., OSYPOV, K. Application of steering filters to localized anisotropic tomography with well data. $80^{\text {th }}$ Annual International Meeting, SEG, Expanded Abstracts, 42864290, 2010b.

CHAKRABORTY, S., L'HEUREUX, E., HARTMAN, K., LI, Q., AHMED, I., JOY, C., BRENDERS, A., SANDSCHAPER, J.R., MITCHELL, S. Thunder Horse Ocean Bottom Nodes acquisition design. 87 th Annual International Meeting, SEG, Expanded Abstracts, 226231, 2017.

FUTTERMAN, W.I. Dispersive body waves. Journal of Geophysical Research, 67, 5279-5291, 1962

HARTMAN, K., CHAKRABORTY, S., NOLTE, B., GOU, W., SUN, Q., CHAZALNOEL, N. Understanding and improving the subsalt image at Thunder Horse, Gulf of 
Mexico. $85^{\text {th }}$ Annual International Meeting, SEG, Expanded Abstracts, 4028-4032, 2015.

JOY, C., REITZ, A., HARTMAN, K. Business impacts of a record breaking $3 \mathrm{D}$ VSP at the Thunder Horse South Field, GoM. 88 $8^{\text {th }}$ Annual International Meeting, SEG, Expanded Abstracts, 5402-5406, 2018.

LEANY, S. Polar Anisotropy from walkaway VSPs. The Leading Edge, Vol. 27, No. 10, pp. 1242-1250, 2008.

LI, Y., LIU, Z., MATEEVA, A., LOPEZ, J. Velocity Analysis and Update with 3D DAS-VSP to Improve Borehole/Surface Seismic Images. $85^{\text {th }}$ Annual International Meeting, SEG, Expanded Abstracts, 52855289, 2015.

PFAU, G., CHEN, R., RAY, A., KAPOOR, J., KOECHNER, B., ALBERTIN, U. Imaging at Thunder Horse. $72^{\text {nd }}$ Annual International Meeting, SEG, Expanded Abstracts, 432-433, 2002.

RAY, A., HORNBY, B., VAN GESTEL, J. Largest 3D VSP in the deep water of the Gulf of Mexico to provide improved imaging in the Thunder Horse South field. $73^{\text {rd }}$ Annual International Meeting, SEG, Expanded Abstracts, 422-425, 2003.

REITZ, A., HARTMAN, K. Demonstrating the value of subsalt 3D VSPs: A case study at Thunder Horse North, Gulf of Mexico. $87^{\text {th }}$ Annual International Meeting, SEG, Expanded Abstracts, 2210-2214, 2017.

VYAS M., DU, X., MOBLEY, E., FLETCHER, R. Methods for computing angle gathers using RTM. $73^{\text {rd }}$ Conference and Exhibition, EAGE, Extended Abstracts, 2011.

WOODWARD, M., NICHOLS, D., ZDRAVEVA, O., WHITFIELD, P., JOHNS, T. A decade of tomography. Geophysics, 73, 5, VE5-VE11, 2008.

ZAMAN, S. O., SARAGOUSSI, E., SOLANO, G. A., ZDRAVEVA, O., HERNANDEZ, V. R. B., LI, Q., CHAKRABORTY, S., JOY, C., HARTMAN, K., REITZ, A. Image Enhancements for Subsalt targets; Thunder Horse case study. $88^{\text {th }}$ Annual International Meeting, SEG, Expanded Abstracts, 4573-4577, 2018.

ZDRAVEVA, O., RAMIREZ, A., CHEN, S., WOODWARD, $M$. Optimized use of 3D VSP data in anisotropic model building for depth imaging. XV International Congress of the Brazilian Geophysical Society, 1823-1827, 2017. 